\title{
Hepatocellular Carcinoma Arising in a Huge Hepatocellular Adenoma with Bone Marrow Metaplasia
}

Hyo Jeong Kang ${ }^{1,2} \cdot$ Hui Jeong Jeong ${ }^{1}$ So-Woon $\mathrm{Kim}^{1} \cdot$ Eunsil $\mathrm{Yu}^{1,3}$ Young-Joo Lee ${ }^{4}$. So Yeon Kim ${ }^{5}$ Jihun Kim ${ }^{1}$

'Departments of Pathology and 2 Physiology, ${ }^{3}$ Asan Liver Center, Departments of ${ }^{4}$ Surgery and ${ }^{5}$ Radiology, Asan Medical Center, University of Ulsan College of Medicine, Seoul, Korea

Received: September 8, 2017

Revised: October 30, 2017

Accepted: November 12, 2017

\section{Corresponding Author}

Jihun Kim, MD, PhD

Department of Pathology, Asan Medical Center,

University of Ulsan College of Medicine, 88

Olympic-ro 43 gil, Songpa-gu, Seoul 05505, Korea

Tel: +82-2-3010-4556

Fax: +82-2-472-7898

E-mail: Jihunkim@amc.seoul.kr
Hepatocellular adenoma (HCA) is the most common type of benign liver tumor, and its major complication is malignant transformation to hepatocellular carcinoma (HCC). Here, we report a case of HCC arising in HCA with bone marrow metaplasia in a 24-year-old Korean woman who presented with abdominal discomfort. A huge liver mass was found on abdominal ultrasonography. She underwent surgical hepatic resection, and the resected specimen was entirely involved by a 20 -cm-sized tumor. Histological review revealed a well differentiated HCC arising from inflammatory HCA with $\beta$-catenin nuclear positivity and bone marrow metaplasia that contained hematopoietic cells. This case was unique because malignant transformation, inflammatory type HCA, $\beta$-catenin nuclear staining, and bone marrow metaplasia were simultaneously observed. Additionally, it should be noted that a large HCA with $\beta$-catenin activation can undergo malignant transformation and should be surgically resected in a timely manner.

Key Words: Carcinoma, hepatocellular; Adenoma, liver cell; Bone marrow metaplasia; $\beta$-catenin
Hepatocellular adenoma (HCA) is the most common benign liver neoplasm that can arise from hepatocytes. Oral contraceptives (OCPs) are the main predisposing factors to the development of HCA; however, other associations, such as obesity, have been more recently recognized. ${ }^{1,2} \mathrm{HCA}$ represents a heterogeneous group of tumors that differ in histology, immunophenotype, molecular pathogenesis, and biological behavior. Based on these factors, HCAs can be sub-classified into the following four major groups: HNF1 $\alpha$-inactivated HCA, $\beta$-catenin activated HCA, inflammatory $\mathrm{HCA}$, and unclassified $\mathrm{HCA}^{2-5}$ Approximately $10 \%$ of inflammatory HCA cases coexist with a $\beta$-catenin mutation. ${ }^{2,4}$ The most important complications of HCAs are hemorrhage and malignant transformation to hepatocellular carcinoma (HCC). ${ }^{6}$ Although the overall risk of malignant transformation has not been well defined, according to previous reports, $4 \%$ $8 \%$ of resected HCA have undergone malignant transformation. ${ }^{6}$ Risk factors for HCC from HCA include male sex, androgen use, large tumor size (> $5 \mathrm{~cm}$ ), and $\beta$-catenin-activated subtype. ${ }^{4,6}$ Bone marrow metaplasia has been very rarely reported in primary liver tumors; indeed, only four such cases have been reported to date. ${ }^{7-10}$ Here, we report for the first time in Korea a case of HCC arising from a $\beta$-catenin-activated inflammatory HCA with bone marrow metaplasia in a young woman who had not taken OCPs.

\section{CASE REPORT}

A 24-year-old woman was admitted to an outside hospital presenting with abdominal discomfort. She was referred to Asan Medical Center and was found to have a huge liver mass that was detected by abdominal ultrasonography. The diagnosis of pre-operative liver biopsy performed by an outside hospital was HCA. Her medical and family histories were unremarkable. She had never taken OCPs and had a body mass index of 21.48 $\mathrm{kg} / \mathrm{m}^{2}$. Hepatitis B surface (HBs) antigen, anti-HBs antibody, and anti-hepatitis $\mathrm{C}$ virus antibody were all negative. A complete blood count and serum chemistry tests, including coagulation and liver function tests, were normal except for elevated 
levels of gamma glutamyl transferase (111 IU/L; normal range, 8 to $61 \mathrm{IU} / \mathrm{L})$. Serum tumor marker of prothrombin induced by vitamin $\mathrm{K}$ absence-II (PIVKA-II) levels were markedly elevated (2,964 mAU/mL; normal range, $\leq 40 \mathrm{mAU} / \mathrm{mL})$. $\alpha$-Fetoprotein levels were slightly elevated $(11.1 \mathrm{ng} / \mathrm{mL}$; normal range, $\leq 7.0$ $\mathrm{ng} / \mathrm{mL}$ ), while those of serum carcinoembryonic antigen (0.9 $\mathrm{ng} / \mathrm{mL}$; normal range, $\leq 5 \mathrm{ng} / \mathrm{mL}$ ) and carbohydrate antigen 19-9 (3.8 U/mL; normal range, $\leq 37 \mathrm{U} / \mathrm{mL})$ were within normal limits. A dynamic liver and pelvis computerized tomography $(\mathrm{CT})$ scan revealed a $20 \mathrm{~cm}$ hypervascular mass occupying segments 4 , 5, and 8 of the liver (Fig. 1A). An enhanced CT image exhibited high attenuation during the arterial phase and decreasing attenuation during the portal and delayed phases. The lesion showed heterogeneous foci of hemorrhage, necrosis, fat, and calcification. An experienced radiologist (S.Y.K.) interpreted the lesion as a huge HCA and suspected a partly malignant transformation. The patient underwent segmentectomy and partial hepatectomy to remove the lesion. Macroscopically, the resected specimen was filled with a well circumscribed $19.9 \times 18.4 \times 7.9-\mathrm{cm}$ solid mass, and its cut surface showed a heterogeneous yellow-brown color, multinodular growth, partly cystic degeneration, hemorrhage, and necrosis (Fig. 1B). Microscopically, the lesion exhibited typical features of a HCA and was characterized by well differentiated hepatocytes arranged in thin cords or sheets with bland cytology and abundant eosinophilic cytoplasm (Fig. 2A). The parenchyma showed dilated sinusoids, occasionally with large aberrant arteries, and multifocal inflammation composed of lymphocytes, neutrophils, and eosinophils (Fig. 2A, B). Immunohistochemical stains of HCA cells showed diffuse positivity of liver fatty acid binding protein, serum amyloid A (Fig. 2C), C- reactive protein (Fig. 2D), and glutamine synthetase (Fig. 2E) antibodies and partial nuclear positivity for $\beta$-catenin antibody (Fig. 2F), suggesting an inflammatory HCA with $\beta$-catenin activation. Although this case showed histologically typical features of inflammatory HCA without histological features of other subtypes, it coexisted with $\beta$-catenin nuclear positivity, suggesting a $\beta$-catenin-activated inflammatory HCA. It was difficult to grossly distinguish the area of malignant transformation. However, multiple malignant foci were found microscopically that occupied less than $10 \%$ of the total tumor area. These malignant foci were all well differentiated HCC that partially resembled the surrounding HCA but also showed nuclear hyperchromasia and pleomorphism and thickened trabeculae (Fig. 2G). Glutamine synthetase showed stronger expression in the HCC area than in the HCA area, and heat shock protein-70 (Fig. 2H) and glypican 3 (Fig. 2I) were positive in the HCC area. As is typical of HCC, reticulin loss (Fig. 2J) and diffuse CD34 expression on sinusoidal cells (Fig. 2K) were observed. The Ki-67 labeling index of the HCC area (15\%) was significantly increased compared with that of the HCA area $(<2 \%)$ (Fig. 2L). Corresponding to visible calcification regions in the $\mathrm{CT}$ scan were areas of bone marrow metaplasia (Fig. 3A) that were characterized by mature lamellar bone forming trabeculae intermingled with fat tissue that contained myeloid and erythroid cells (Fig. 3B, C). The patient was discharged 10 days after surgery and showed no evidence of recurrence at the last follow up visit 23 months after surgery.

This study was approved by the Institutional Review Board of Asan Medical Center with a waiver of informed consent (IRB No.2017-0679).
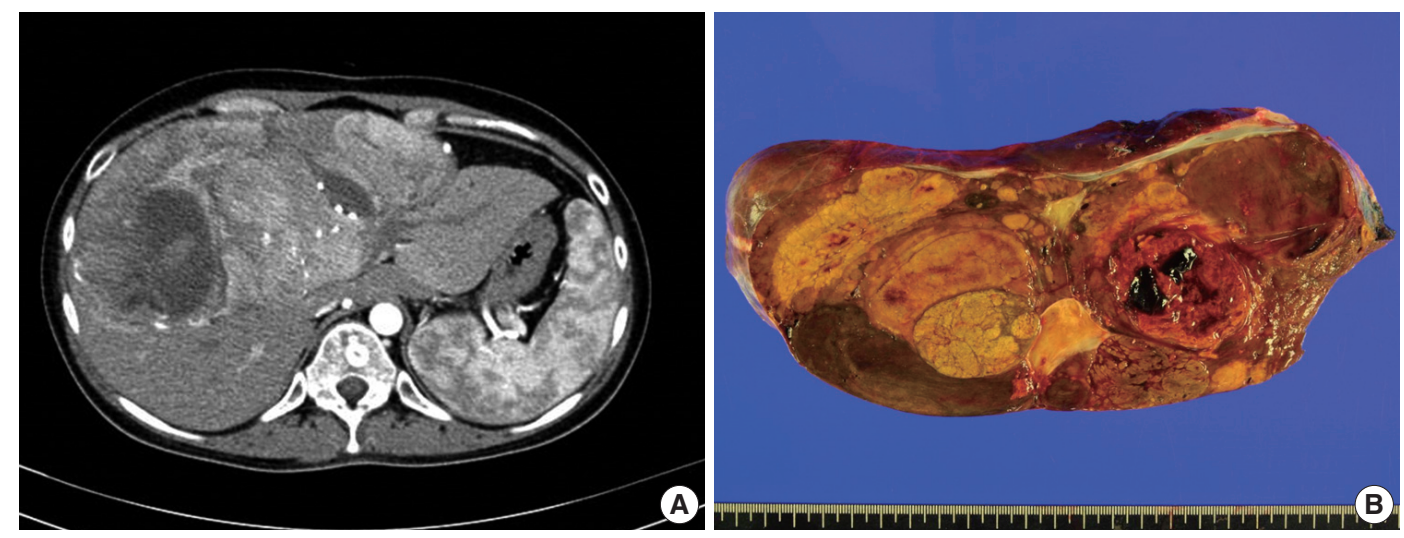

Fig. 1. Imaging and gross pathological findings. (A) Dynamic liver and pelvis computed tomography shows an enlarged liver with a huge, well-defined, hypervascular mass in segments 4, 5, and 8 of the liver during arterial phase. (B) The surface of a slice from a surgically resected specimen reveals a heterogeneously yellow-brown color and multinodular growth with partly cystic degeneration that contained hemorrahge and necrosis. 


\section{DISCUSSION}

HCA is an uncommon benign liver neoplasm composed of he- patocytes and typically develop in a non-cirrhotic liver. It primarily occurs in young to middle-aged women with a history of OCP use. Estrogen or exogenous androgen use for medical purposes
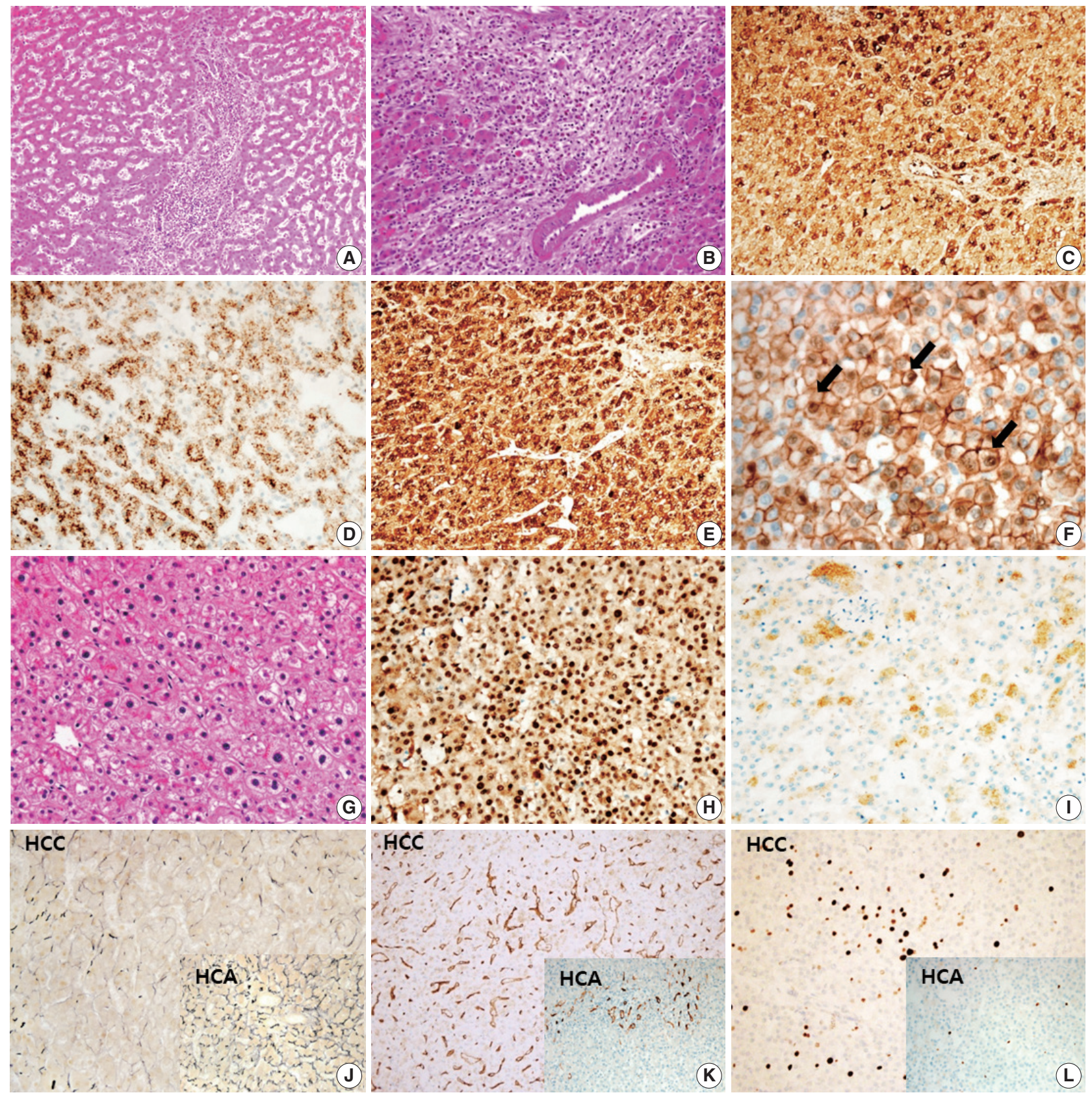

Fig. 2. Microscopic and immunohistochemical findings of hepatocellular adenoma $(H C A)$, inflammatory type with $\beta$-catenin activation (A-F) and hepatocellular carcinoma (HCC) $(\mathrm{G}-\mathrm{L})$. (A) Dilated sinusoids and intratumoral inflammation in the portal tract-like areas are observed in the parenchyma. (B) Large aberrant arteries with infiltration of variable inflammatory cells including lymphocytes, neutrophils, and eosinophils are occasionally observed. Tumor cells show diffuse cytoplasmic expression of serum amyloid A (C), C-reactive protein (D), and glutamine synthetase (E). (F) Nuclear $\beta$-catenin expression (arrows) is observed in several tumor cells. (G) Nuclear atypia and thick trabeculae are observed in the HCC component. (H) Heat shock protein-70 is strongly and diffusely positive. (I) Glypican 3 is partially positive. (J) Loss of reticulin is observed in the HCC component, whereas reticulin staining is preserved in the HCA (inset). (K) CD34 expression on sinusoidal cells is strong and diffuse in HCC but is patchy in HCA (inset). (L) The Ki-67 labeling index of the HCC area is significantly increased compared with that of HCA (inset). 

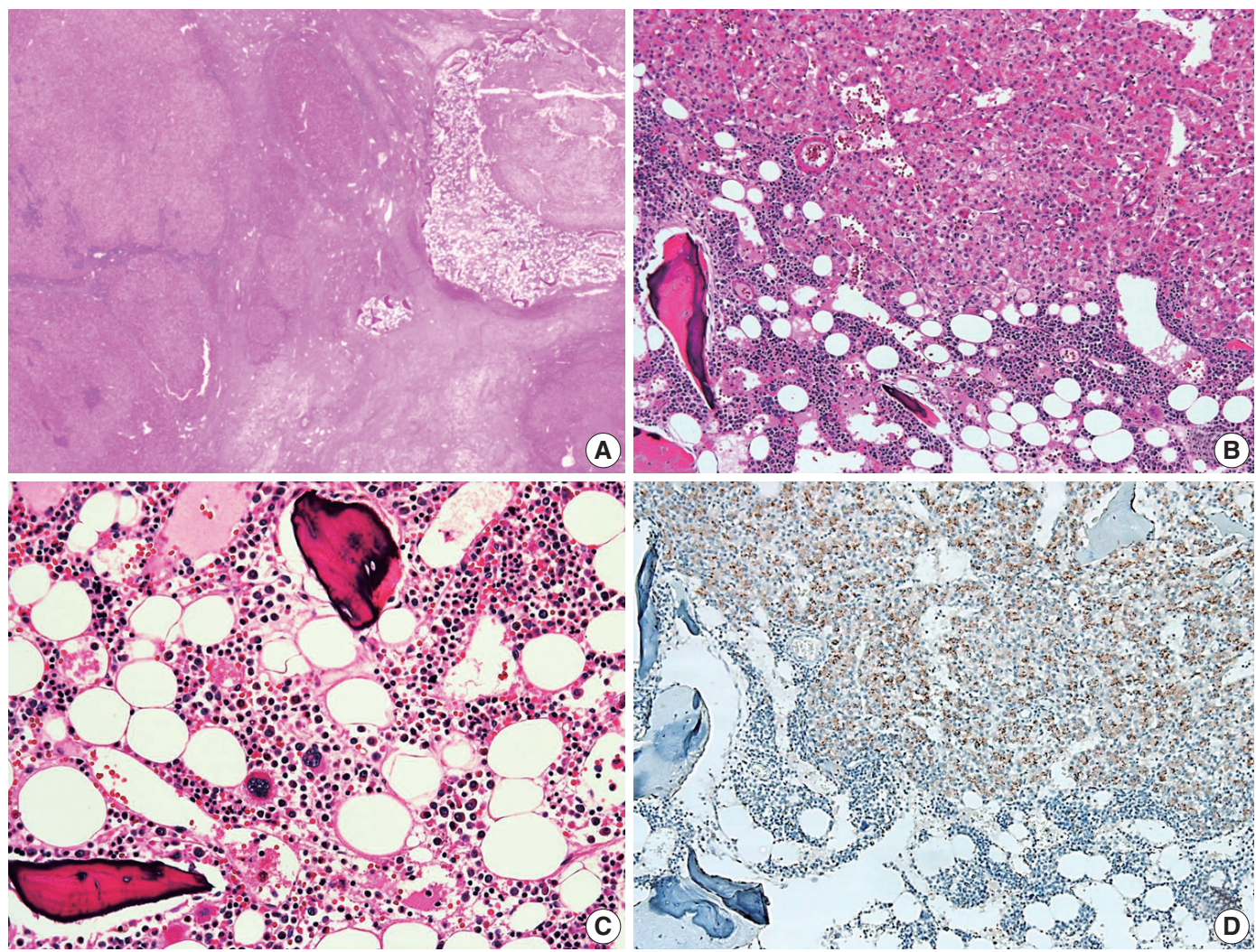

Fig. 3. Histological findings of bone marrow metaplasia. (A) Bone marrow metaplasia is observed in the inner hepatocellular adenoma. (B, C) Bone marrow metaplasia is characterized by mature lamellar bone that formed trabeculae intermingled with fat tissue containing erythroblasts, myeloblasts, and megakaryocytes. (D) Tumor cells near the bone marrow metaplasia shows positivity for epithelial cell adhesion molecule immunohistochemical staining.

is also a recognized risk factor for HCA. Additionally, rarely HCA is found in children and is association with other conditions such as glycogen storage disease, galactosemia, tyrosinemia, familial polyposis coli, Fanconi anemia, and hepatic iron overload with $\beta$-thalassemia. ${ }^{11,12}$ It has recently been reported that the incidence of $\mathrm{HCA}$ is increasing in males and non-OCP individuals. Additionally, there is a trend towards increasing cases of multiple HCAs. This may primarily be a consequence of the higher prevalence of obesity and metabolic syndrome, rather than an effect of OCPs, which have been considered to be the main cause for the development of HCA. ${ }^{1,13,14}$ Interestingly, the woman with HCA in this study had a huge mass at a young age, and she showed no history of OCP use or genetically determined metabolic disease.

Malignant transformation of HCA is the main reason for surgical treatment. It is important to identify factors that increase the risk of malignancy. However, HCA is difficult to distinguish from HCC because of its similar imaging characteristics and histopathological features. ${ }^{6}$ Several studies have indicated that high risk groups of patients having an increased risk of malignant alteration from HCA display larger tumors $(>5 \mathrm{~cm})$, have a history of androgen or anabolic steroid intake, are male, and are patients with glycogen storage disease. ${ }^{12,15-19}$ HCA has recently been roughly classified into four subgroups according to genotype-phenotype based analysis. Moreover, $\beta$-catenin-activated HCA represents a well-known factor for an increased risk of malignant transformation compared with the other subtypes. ${ }^{17}$ In our present case, although the patient was a young woman who had only abdominal discomfort without any symptoms related to malignancy the large tumor size and high level of PIVKA-II could predict the possibility of malignant transformation from HCA. Therefore, a rapid assessment of mass removal was performed to prevent intraperitoneal hemorrhage, rupture, malignant progression, or metastasis. Within the surgically resected specimen, partially malignant transformation was confirmed by histological and immunohistochemical examination. Nuclear accumulation of $\beta$-catenin antibody was detected in areas corresponding to malignant foci by immunohistochemistry, which was consistent with an association with malignant transformation in HCA.

A notable feature of this case was the presence of bone marrow 
Table 1. Overview of cases of HCA and HCC with bone marrow metaplasia

\begin{tabular}{|c|c|c|c|c|c|c|c|c|}
\hline \multirow{2}{*}{ Study } & \multirow{2}{*}{ Year } & \multirow{2}{*}{ Age (yr) } & \multirow{2}{*}{ Sex } & \multirow{2}{*}{ Diagnosis } & \multirow{2}{*}{$\begin{array}{l}\text { Associated } \\
\text { disease }\end{array}$} & \multirow{2}{*}{ Size $(\mathrm{cm})$} & \multicolumn{2}{|c|}{ Bone marrow metaplasia } \\
\hline & & & & & & & Fat component & Hematopoietic cells \\
\hline Moriura et al. ${ }^{9}$ & 1996 & 34 & Male & $\mathrm{HCA}(\times 2)$ & GSD type 1a & Up to 12 & Present & Absent \\
\hline Ramacciato et al..$^{10}$ & 2006 & 58 & Male & $\mathrm{HCA}$ & Absent & 14 & Present & Present \\
\hline Copin et al. ${ }^{7}$ & 2015 & 42 & Female & $\mathrm{HCC}$ & Absent & No mention & Present & Present \\
\hline Iguchi et al. ${ }^{8}$ & 2016 & 46 & Female & HCC arising in HCA (multiple) & GSD type I & Up to 10 & Present & Present \\
\hline Present case & 2017 & 24 & Female & $\mathrm{HCC}$ arising in $\mathrm{HCA}$ & Absent & 20 & Present & Present \\
\hline
\end{tabular}

HCA, hepatocellular adenoma; HCC, hepatocellular carcinoma; GSD, glycogen storage disease.

metaplasia in the inner HCA. Bone or osteoid formation has been found in special types of primary liver tumors, especially hepatoblastoma. However, bone marrow metaplasia with hematopoietic cells is extremely rare in primary liver tumors in adults, especially in cases of HCA or HCC. Only four cases have been reported in the English literature in which areas of bone marrow metaplasia were found in HCC or HCA (Table 1). According to a review of the literature, this represents the first case report of HCC arising from HCA with complete bone marrow metaplasia consisting of both fat components and hematopoietic cells in an adult younger than 30 years old. It has been previously demonstrated that bone marrow-derived stem cells can differentiate into hepatic progenitor cells that may play a role in liver tumor development. ${ }^{10,20}$ In this present case, hepatic progenitor cells with epithelial cell adhesion molecule expression (Fig. 3D) were observed around bone marrow metaplasia but were less frequent or not detected in areas far from bone marrow metaplasia.

In conclusion, we report a case of HCC arising from inflammatory HCA with $\beta$-catenin activation. Two important points of this case are that malignant transformation in huge HCA can occur in a young woman without risk factors such as use of OCP or genetic metabolic diseases and that complete bone marrow metaplasia can occur in HCA. This case report emphasizes that a large HCA can show malignant transformation, even in a young woman, and that these tumors should be promptly, surgically resected.

\section{ORCID}

Jihun Kim: https://orcid.org/0000-0002-8694-4365

\section{Conflicts of Interest}

No potential conflict of interest relevant to this article was reported.

\section{REFERENCES}

1. Chang CY, Hernandez-Prera JC, Roayaie S, Schwartz M, Thung
SN. Changing epidemiology of hepatocellular adenoma in the United States: review of the literature. Int J Hepatol 2013; 2013: 604860.

2. Bioulac-Sage P, Sempoux C, Balabaud C. Hepatocellular adenoma: classification, variants and clinical relevance. Semin Diagn Pathol 2017; 34: 112-25.

3. Bioulac-Sage P, Balabaud C, Zucman-Rossi J. Subtype classification of hepatocellular adenoma. Dig Surg 2010; 27: 39-45.

4. Margolskee E, Bao F, de Gonzalez AK, et al. Hepatocellular adenoma classification: a comparative evaluation of immunohistochemistry and targeted mutational analysis. Diagn Pathol 2016; 11: 27.

5. Chu HH, Moon WS. $\beta$-catenin activated hepatocellular adenoma. Clin Mol Hepatol 2013; 19: 185-9.

6. Stoot JH, Coelen RJ, De Jong MC, Dejong CH. Malignant transformation of hepatocellular adenomas into hepatocellular carcinomas: a systematic review including more than 1600 adenoma cases. HPB (Oxford) 2010; 12: 509-22.

7. Copin P, Ronot M, Vilgrain V. Hepatocellular carcinoma with osseous metaplasia and bone marrow elements. Clin Gastroenterol Hepatol 2015; 13: e26-7.

8. Iguchi T, Yamagata M, Sonoda T, et al. Malignant transformation of hepatocellular adenoma with bone marrow metaplasia arising in glycogen storage disease type I: a case report. Mol Clin Oncol 2016; 5: 599-603.

9. Moriura S, Kuroda M, Kimura A, et al. Case report: hepatic adenoma with bone marrow metaplasia in a patient with glycogen storage disease type 1a. J Gastroenterol Hepatol 1996; 11: 556-9.

10. Ramacciato G, Nigri GR, Aurello P, et al. Giant hepatic adenoma with bone marrow metaplasia not associated with oral contraceptive intake. World J Surg Oncol 2006; 4: 58.

11. Vaithianathan R, Philipchandran, Selvambigai G, Jayaganesh P. Spontaneous hepatocellular adenoma in paediatric age group: case report. J Clin Diagn Res 2013; 7: 2962-3.

12. Velazquez I, Alter BP. Androgens and liver tumors: Fanconi's anemia and non-Fanconi's conditions. Am J Hematol 2004; 77: 257-67.

13. Stueck AE, Qu Z, Huang MA, Campreciós G, Ferrell LD, Thung $\mathrm{SN}$. Hepatocellular carcinoma arising in an HNF-1alpha-mutated 
adenoma in a 23-year-old woman with maturity-onset diabetes of the young: a case report. Semin Liver Dis 2015; 35: 444-9.

14. Hirata E, Shimizu S, Umeda S, et al. Hepatocyte nuclear factor 1alphainactivated hepatocellular adenomatosis in a patient with maturityonset diabetes of the young type 3: case report and literature review. Nihon Shokakibyo Gakkai Zasshi 2015; 112: 1696-704.

15. Dokmak S, Paradis V, Vilgrain V, et al. A single-center surgical experience of 122 patients with single and multiple hepatocellular adenomas. Gastroenterology 2009; 137: 1698-705.

16. Gorayski P, Thompson CH, Subhash HS, Thomas AC. Hepatocellular carcinoma associated with recreational anabolic steroid use. Br J Sports Med 2008; 42: 74-5.
17. Zucman-Rossi J, Jeannot E, Nhieu JT, et al. Genotype-phenotype correlation in hepatocellular adenoma: new classification and relationship with HCC. Hepatology 2006; 43: 515-24.

18. Franco LM, Krishnamurthy V, Bali D, et al. Hepatocellular carcinoma in glycogen storage disease type Ia: a case series. J Inherit Metab Dis 2005 ; 28 : 153-62

19. Terkivatan T, de Wilt JH, de Man RA, et al. Indications and longterm outcome of treatment for benign hepatic tumors: a critical appraisal. Arch Surg 2001; 136: 1033-8.

20. Herzog EL, Chai L, Krause DS. Plasticity of marrow-derived stem cells. Blood 2003; 102: 3483-93. 